\title{
A Fourth-Order Finite-Difference Approximation for the Fixed Membrane Eigenproblem*
}

\author{
By J. R. Kuttler
}

\begin{abstract}
The fixed membrane problem $\Delta u+\lambda u=0$ in $\Omega, u=0$ on $\partial \Omega$, for a bounded region $\Omega$ of the plane, is approximated by a finite-difference scheme whose matrix is monotone. By an extension of previous methods for schemes with matrices of positive type, $O\left(h^{4}\right)$ convergence is shown for the approximating eigenvalues and eigenfunctions, where $h$ is the mesh width. An application to an approximation of the forced vibration problem $\Delta u+q u=f$ in $\Omega, u=0$ in $\partial \Omega$, is also given.
\end{abstract}

1. Introduction. Let $\Omega$ be a bounded region of the plane with smooth boundary $\partial \Omega$. We consider the fixed membrane problem

$$
\Delta u(x)+\lambda u(x)=0, \quad x \in \Omega, \quad u(x)=0, \quad x \in \partial \Omega,
$$

where $\Delta$ is the Laplacian. In [6], this problem was approximated by difference schemes which were of positive type in the interior of the region. Here, we consider a difference scheme for (1.1) which is only monotone. However, by appropriate modifications of the techniques of [6], we can prove that this scheme yields $O\left(h^{4}\right)$ approximations to the eigenvalues and eigenvectors of (1.1). The principal result is Theorem 8.1. An application to a forced vibration problem is also given in Section 9.

2. The Difference Scheme. Let $h>0$ be given and define the mesh $S_{h}$ by

$$
\{(i h, j h): i, j \text { are integers }\} \text {. }
$$

Points $x, y \in S_{h}$ will be called nearest neighbors if $|x-y|=h$, where we write

$$
|x-y|=\left(\left(x_{1}-y_{1}\right)^{2}+\left(x_{2}-y_{2}\right)^{2}\right)^{1 / 2} .
$$

Let $\Omega_{h}^{(3)}$ be the set of points in $S_{h} \cap \Omega$ having at least one nearest neighbor not in $\Omega$. One such point might be $x=\left(x_{1}, x_{2}\right)$ with $\left(x_{1}-\alpha h, x_{2}\right),\left(x_{1}, x_{2}-\beta h\right) \in \partial \Omega$ for $0<\alpha, \beta \leqq 2$. If $\left(x_{1}+h, x_{2}\right),\left(x_{1}+2 h, x_{2}\right),\left(x_{1}, x_{2}+h\right),\left(x_{1}, x_{2}+2 h\right) \in \Omega$, we define

Received September 11, 1969, revised September 9, 1970.

AMS 1970 subject classifications. Primary 35P15, 35J05, 65N10, 65N15, 65N25; Secondary 15A48, $15 \mathrm{~A} 42$.

Key words and phrases. Finite-differences, membrane, fixed membrane, eigenvalues, elliptic partial differential equations, monotone matrices, forced vibration problem, discrete Green's function.

* This work was supported by the Department of the Navy, Bureau of Naval Weapons, under Contract No. w-62-0604-c. 


$$
\begin{aligned}
h^{2} l_{h}(x, y) & =\frac{3-\alpha}{\alpha}+\frac{3-\beta}{\beta}, & & y=x, \\
& =-\frac{2(2-\alpha)}{1+\alpha}, & & y=\left(x_{1}+h, x_{2}\right), \\
& =-\frac{2(2-\beta)}{1+\beta}, & & y=\left(x_{1}, x_{2}+h\right), \\
& =\frac{1-\alpha}{2+\alpha}, & & y=\left(x_{1}+2 h, x_{2}\right), \\
& =\frac{1-\beta}{2+\beta}, & & y=\left(x_{1}, x_{2}+2 h\right), \\
& =0, & & \text { otherwise. }
\end{aligned}
$$

Similar formulas apply at other points of $\Omega_{h}^{(3)}$. One special case may arise, as shown in Fig. 1, where $\left(x_{1}, x_{2}+h\right),\left(x_{1}, x_{2}+2 h\right)$ do not lie in $\Omega$.

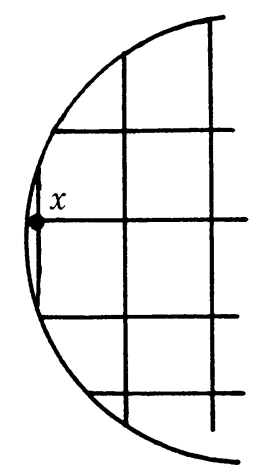

FIGURE 1

In such a case $x$ would be excluded from the difference scheme altogether and the point $\left(x_{1}+h, x_{2}\right)$ would be added to $\Omega_{h}^{(3)}$. For the new point, formula (2.1) would be used with $1<\alpha \leqq 2$. If $\partial \Omega$ has bounded curvature and $h$ is sufficiently small, there will be no difficulty with the new point.

Next, let $\Omega_{h}^{(2)}$ be those points of $S_{h} \cap \Omega$, not in $\Omega_{h}^{(3)}$ or excluded, which have a nearest neighbor in $\Omega_{h}^{(3)}$. For $x \in \Omega_{h}^{(2)}$ define

$$
\begin{aligned}
h^{2} l_{h}(x, y) & =4, & & y=x, \\
& =-1, & & |x-y|=h, \quad y \in S_{h}, \\
& =0, & & \text { otherwise. }
\end{aligned}
$$

Finally, let $\Omega_{h}^{\prime}$ be those points of $S_{h} \cap \Omega$ not in $\Omega_{h}^{(2)} \cup \Omega_{h}^{(3)}$ or excluded. For $x \in \Omega_{h}^{\prime}$ define

$$
\begin{aligned}
& h^{2} l_{\Lambda}(x, y)=5, \quad y=x, \\
& =-\frac{4}{3}, \quad|x-y|=h, \quad y \in S_{h}, \\
& =\frac{1}{12}, \quad|x-y|=2 h, \quad y \in S_{h} \text {, } \\
& =0 \text {, otherwise. }
\end{aligned}
$$


Let $\Omega_{h}=\Omega_{h}^{\prime} \cup \Omega_{h}^{(2)} \cup \Omega_{h}^{(3)}$. We approximate the Laplacian of a function $u$ vanishing on $\partial \Omega$ by

$$
-\Delta_{h} u(x)=\sum_{y \in \Omega_{h}} l_{h}(x, y) u(y), \quad x \in \Omega_{h} .
$$

Let us agree to use $C$ as a generic constant, whose value may change at each usage, but which is always independent of $h$. Then, if also $u \in C^{6}(\bar{\Omega})(u$ has continuous sixth derivatives on the closure of $\Omega$ ), it can be seen from Taylor series expansions that

$$
\begin{aligned}
\left|\Delta u(x)-\Delta_{h} u(x)\right| & \leqq C h^{4}, \quad x \in \Omega_{h}^{\prime}, \\
& \leqq C h^{2}, \quad x \in \Omega_{h}^{(2)} \cup \Omega_{h}^{(3)} .
\end{aligned}
$$

Bramble and Hubbard used $\Delta_{h}$ in [2] in approximating the Dirichlet problem for Poisson's equation.

Our difference scheme approximating (1.1) is

$$
\Delta_{h} U_{h}(x)+\lambda_{h} U_{h}(x)=0, \quad x \in \Omega_{h} .
$$

Problem (2.6) is equivalent to finding the eigenvalues and eigenvectors of the matrix $\left[l_{h}(x, y)\right]_{x, y \in \Omega_{h}}$. In the next section, we develop some tools to use in studying this matrix which, however, have some independent interest.

3. Monotone Matrices. Let $A=\left(a_{i j}\right)$ be an $n \times n$ matrix. We say $A \geqq 0$ if each $a_{i j} \geqq 0$ and $A \leqq B$ if $B-A \geqq 0$. The matrix $A$ is monotone if $A x \geqq 0$ implies $x \geqq 0$ for all $x$. Thus, $A$ is monotone if and only if $A^{-1}$ exists and $A^{-1} \geqq 0$. An easily recognized type of monotone matrix is a matrix of positive type. The matrix $A$ is of positive type if $A$ is indecomposable, the diagonal of $A$ is positive, the off-diagonal elements negative, and the row sums are nonnegative with at least one strictly positive. The following theorem is due to Price [8]:

THEOREM 3.1. $A$ is monotone if and only if there exists $M$ monotone such that

(i) $M^{-1}(M-A) \geqq 0$,

(ii) $\rho\left(M^{-1}(M-A)\right)<1$.

Here $\rho$ denotes spectral radius, the maximum of the moduli of the eigenvalues. Here and in the corollaries, the "only if" part is trivial: take $M=A$. This theorem generalizes Theorem 2.7 of Bramble and Hubbard [2]. There are a number of important corollaries:

COROLlary 3.2. $A$ is monotone if and only if there exists $M$ monotone such that

(i) $M \geqq A$,

(ii) $\rho\left(M^{-1}(M-A)\right)<1$.

Corollary 3.3. $A$ is monotone if and only if there exists $M$ monotone and $x>0$ such that

(i) $M \geqq A$,

(ii) $A x>0$.

Proof. By the Gerschgorin circle theorem (see [7, p. 152]),

since

$$
\rho\left(M^{-1}(M-A)\right) \leqq \max _{i}\left[M^{-1}(M-A) x\right]_{i} / x_{i}<1,
$$

$$
0 \leqq\left[M^{-1}(M-A) x\right]_{i}=x_{i}-\left[M^{-1} A x\right]_{i}<x_{i},
$$

because $A x>0, M^{-1} \geqq 0$ and no row of $M^{-1}$ can be all zero. 
COROLlaRY 3.4. $A$ is monotone if and only if there exists $M$ monotone and $x \geqq 0$ such that

(i) $M \geqq A$,

(ii) $A x>0$.

Proof. Let $\delta=\min _{i}[A x]_{i}>0$ and let $\epsilon=\delta /\left(2 \max _{i} \sum_{i}\left|a_{i j}\right|\right)$. Then $x+\epsilon>0$ and $A(x+\epsilon)>0$, so the hypotheses of Corollary 3.4 are satisfied.

COROLlary 3.5. $A$ is monotone if and only if there exist $M_{1}, M_{2}$ monotone such that

$$
M_{1} \leqq A \leqq M_{2} \text {. }
$$

Proof. Let $x$ be such that $M_{1} x$ is the vector with all components 1 . Since $M_{1}$ is monotone, $x$ exists and $x \geqq 0$. Also, $A x \geqq M_{1} x>0$, so the hypotheses of Corollary 3.4 are satisfied.

COROLlaRY 3.6. $A$ is monotone if there is $\alpha>0$ such that $A+\alpha I$ is monotone and every eigenvalue $\lambda$ of $A$ has positive real part.

Proof. Apply Corollary 3.2. We need only show $\rho\left((A+\alpha I)^{-1}\right)<\alpha^{-1}$. But $\rho\left((A+\alpha)^{-1}\right)=1 / \min _{\lambda}|\alpha+\lambda|$, where $\lambda$ runs over the eigenvalues of $A$.

At this time, we also note the following:

LEMMA 3.7. If the partitioned matrix

$$
\left[\begin{array}{ll}
A & B \\
C & D
\end{array}\right]
$$

with $A$ invertible has inverse

$$
\left[\begin{array}{ll}
W & X \\
Y & Z
\end{array}\right]
$$

then $W-A^{-1}=-X C A^{-1}$. In particular, if $X \geqq 0, A^{-1} \geqq 0, C \leqq 0$, then $A^{-1} \leqq W$.

Proof. Since

$$
\left[\begin{array}{ll}
W & X \\
Y & Z
\end{array}\right]\left[\begin{array}{ll}
A & B \\
C & D
\end{array}\right]=\left[\begin{array}{ll}
I & 0 \\
0 & I
\end{array}\right],
$$

we have $W A+X C=I$. Multiply on the right by $A^{-1}$.

4. Discrete Green's Functions. The main tools in our investigations will be discrete analogues of Green's function. These are inverses of matrices related to $\left[h^{2} l_{h}(x, y)\right]_{x, y \in \Omega_{k}}$ and their nonnegativity is crucial to the investigation. This will be established, using results of the previous section.

We define then

(4.1) $-\Delta_{h, x} g_{h}(x, y)=h^{-2} \delta(x, y), x \in \Omega_{h}^{\prime} \cup \Omega_{h}^{(2)}, g_{h}(x, y)=\delta(x, y), x \in \Omega_{h}^{(3)}$,

for all $y \in \Omega_{h}$. This is the discrete Green's function considered by Bramble and Hubbard in [2, Eq. (4.5)]. From (4.1), we see that the matrix $\left[g_{h}(x, y)\right]_{x, y \in 0_{h}}$ is the inverse of the partitioned matrix

$$
\mathfrak{M} \equiv\left[\begin{array}{ll}
A & B \\
0 & I
\end{array}\right],
$$


where $A=\left[h^{2} l_{h}(x, y)\right]_{x, y \in \Omega_{h^{\prime}} \cup \Omega_{h}(s)}, B=\left[h^{2} l_{h}(x, y)\right]_{x \in \Omega_{\boldsymbol{h}^{\prime}} \cup \Omega_{\mathbf{h}}(s), y \in \Omega_{h}(s)}$, and $I$ is the identity on $\Omega_{h}^{(3)} \times \Omega_{h}^{(3)}$. It also follows from Lemma 3.7 that the matrix $\left[g_{h}(x, y)\right]_{x, y \in Q_{A}^{\prime} \cup \Omega_{k}(s)}$ is the inverse of $A$. In [2], it was shown that

$$
g_{h}(x, y) \geqq 0, \quad x, y \in \Omega_{h},
$$

i.e., $\mathfrak{M}$ is monotone. Since $g_{h}$ is the inverse, it follows that, for any function $W$ defined on $\Omega_{h}$, all $x \in \Omega_{h}$,

$$
W(x)=h^{2} \sum_{y \in \Omega_{h}^{\prime} \cup \Omega_{h}(s)} g_{h}(x, y)\left[-\Delta_{h} W(y)\right]+\sum_{y \in \Omega_{h}(s)} g_{h}(x, y) W(y) .
$$

This is analogous to Poisson's formula. In [2], the following properties were proved of $g_{h}$ :

$$
\begin{gathered}
\sum_{y \in \Omega_{h}(s)} g_{h}(x, y) \leqq 1, \\
\sum_{y \in \Omega_{h}(z)} g_{h}(x, y) \leqq C, \\
h^{2} \sum_{y \in \Omega_{h}} g_{h}(x, y) \leqq C,
\end{gathered}
$$

for all $x \in \Omega_{h}$. Using these in (4.3), we have the inequality

$$
\max _{\Omega_{A}}|W| \leqq C\left[\max _{\Omega_{h^{\prime}}}\left|\Delta_{h} W\right|+h^{2} \max _{\Omega_{h}(s)}\left|\Delta_{h} W\right|\right]+\max _{\Omega_{h}(s)}|W|
$$

Now, on $\Omega_{h}^{(3)}$, we have

$$
W(x)=\left[-h^{2} \Delta_{h} W(x)-h^{2} \sum_{y \in \Omega_{h} ; y \neq x} l_{h}(x, y) W(y)\right] / h^{2} l_{h}(x, x),
$$

and from this and (2.1), we see that

$$
\max _{\Omega_{h}(0)}|W| \leqq C h^{2} \max _{\Omega_{h}(s)}\left|\Delta_{h} W\right|+\theta \max _{\Omega_{A}}|W|,
$$

where

$$
\theta=\max _{x \in \Omega_{h}(s)} \sum_{y \in \Omega_{h} ; y ; x}\left|l_{h}(x, y)\right| / l_{h}(x, x)<1 .
$$

Putting (4.8) into (4.7) and rearranging, we have

$$
\max _{\Omega_{h}}|W| \leqq C\left[\max _{\Omega_{h^{\prime}}}\left|\Delta_{h} W\right|+h^{2} \max _{\Omega_{h}(s) \cup \Omega_{h}(s)}\left|\Delta_{h} W\right|\right] .
$$

Let us now use (4.7) to estimate $W=\Phi_{h}-\varphi$ where $\varphi$ is the torsion function defined by $\Delta \varphi=-1$ on $\Omega, \varphi=0$ on $\partial \Omega$ and $\Phi_{h}(x)=h^{2} \sum_{\nu \in \Omega_{h}} g_{h}(x, y)$, which satisfies $\Delta_{h} \Phi_{h}=-1$ on $\Omega_{h}^{\prime} \cup \Omega_{h}^{(2)}$. If $\partial \Omega$ is sufficiently smooth, $\varphi$ satisfies (2.5) and we see from (4.7) that

$$
\max _{\Omega_{A}}\left|\Phi_{h}-\varphi\right| \leqq C h^{4}+\max _{\Omega_{h}(s)}\left|\Phi_{h}-\varphi\right| \leqq C h^{4}+\max _{\Omega_{A}(s)}\left|\Phi_{h}\right|+\max _{\Omega_{h}(s)}|\varphi| .
$$

Now, $\varphi=0$ on $\partial \Omega$, so $|\varphi(x)| \leqq C h$ for $|x-\partial \Omega|=\min _{\nu \in \partial \Omega}|x-y| \leqq C h$. Also, $\Phi_{h}=h^{2}$ on $\Omega_{h}^{(3)}$ by definition. Hence,

$$
\left|\Phi_{h}(x)\right| \leqq|\varphi(x)|+\max _{\Omega_{h}}\left|\Phi_{h}-\varphi\right| \leqq C h
$$


for $|x-\partial \Omega| \leqq C h$, i.e.,

$$
h^{2} \sum_{\nu \in \Omega_{h}} g_{h}(x, y) \leqq C h .
$$

Next, we consider the function

$$
f_{h}(x, y)=C_{1}-C_{2} \log \left(|x-y|^{2}+h^{2}\right) .
$$

It is easily verified that

$$
\begin{array}{ll}
-\Delta_{h, x} f_{h}(x, y) \geqq 0, & x \in \Omega_{h}^{\prime} \cup \Omega_{h}^{(2)}, \quad y \neq x, \\
-\Delta_{h, x} f_{h}(x, y) \geqq h^{-2}, & x \in \Omega_{h}^{\prime} \cup \Omega_{h}^{(2)}, \quad y=x,
\end{array}
$$

provided $C_{2} \geqq \frac{1}{4} \log 2$. If we choose

$$
C_{1}=C_{2} \max _{x, y \in \Omega_{h}} \log \left(|x-y|^{2}+h^{2}\right),
$$

then $f_{h}(x, y) \geqq 0$ for $x, y \in \Omega$. Thus, we see that

$$
\mathfrak{M}\left(f_{h}-g_{h}\right) \geqq 0,
$$

and, since $\mathfrak{M}$ is monotone,

$$
0 \leqq g_{h}(x, y) \leqq C_{1}-C_{2} \log \left(|x-y|^{2}+h^{2}\right) .
$$

Analogous inequalities to (4.11) are proved by Bramble and Thomée in [3] for discrete Green's functions of positive-type operators. Here, we see monotonicity was sufficient. An easy consequence of (4.11) is

$$
h^{2} \sum_{y \in \Omega_{\Lambda}}\left[g_{h}(x, y)\right]^{2} \leqq C .
$$

5. More Inequalities for Green's Functions. This section will be devoted to derivations of some inequalities of more difficulty than those of the previous section.

Recall that $\$ S=\left[g_{h}(x, y)\right]_{x, y \in \Omega_{\mathrm{a}}}$ is the inverse of $\left[h^{2} l_{h}(x, y)\right]_{x, y \in \Omega_{h}}$.

The inequality which we next wish to derive is

$$
\sum_{y \in \Omega_{h}^{\prime}} g_{h}(x, y) \leqq C
$$

for all $x \in \Omega_{h}$, where $\Omega_{h}^{\prime \prime}=\left\{x \in \Omega_{h}^{\prime}: l_{h}(x, y) \neq 0\right.$ for some $\left.y \in \Omega_{h}^{(2)} \cup \Omega_{h}^{(3)}\right\}$. The method of proof is the matrix splitting technique employed by Bramble and Hubbard in [2]. The analysis which follows is regrettably detailed.

Let us write

$$
\text { (S) }=\left[I-H_{1}-H_{2}\right]^{-1} \tilde{D}^{-1},
$$

where $\tilde{D}$ is the diagonal matrix with

$$
\begin{aligned}
\tilde{d}_{x x}^{-1} & =1, & & x \in \Omega_{h}^{(3)}, \\
& =\frac{1}{4}, & & x \in \Omega_{h}^{(2)}, \\
& =\frac{1}{5}, & & x \in \Omega_{h}^{\prime},
\end{aligned}
$$


and

$$
\begin{aligned}
& {\left[H_{1}\right]_{x y}=\frac{2}{15}, \quad x \in \Omega_{h}^{\prime}, \quad|x-y|=h,} \\
& =\frac{1}{8}, \quad x \in \Omega_{h}^{(2)},|x-y|=h, \\
& =0 \text {, otherwise, } \\
& {\left[H_{2}\right]_{x y}=\frac{2}{15}, \quad x \in \Omega_{h}^{\prime}, \quad|x-y|=h,} \\
& =-\frac{1}{60}, \quad x \in \Omega_{h}^{\prime}, \quad|x-y|=2 h, \\
& =\frac{1}{8}, \quad x \in \Omega_{h}^{(2)}, \quad|x-y|=h, \\
& =0, \quad \text { otherwise. }
\end{aligned}
$$

Let us define the diagonal matrix $D$ by

$$
\begin{aligned}
\left(d_{x x}\right)^{-1}=\sum_{\nu \in \Omega_{h}}\left(I-H_{1}\right)_{x y} & =\frac{7}{15}, & & x \in \Omega_{h}^{\prime}, \\
& =\frac{1}{2}, & & x \in \Omega_{h}^{(2)}, \\
& =1, & & x \in \Omega_{h}^{(3)},
\end{aligned}
$$

so that $D\left(I-H_{1}\right)$ has row sums one, i.e.,

$$
\sum_{y \in \Omega_{\star}}\left[D\left(I-H_{1}\right)\right]_{x y}=\sum_{y \in \Omega_{\sharp}}\left[\left(I-H_{1}\right)^{-1} D^{-1}\right]_{x y}=1 .
$$

We write $\left[I-H_{1}-H_{2}\right]=\left[D^{-1}(I-H)\right]\left[D\left(I-H_{1}\right)\right]$, where $H=D H_{2}\left(I-H_{1}\right)^{-1} D^{-1}$. Thus, by (5.3),

$$
\begin{aligned}
\sum_{y \in \Omega_{h}}\left[D^{-1}(I-H)\right]_{x y} & =\sum_{\nu, z \in \Omega_{\Lambda}}\left[D^{-1}(I-H)\right]_{x y}\left[D\left(I-H_{1}\right)\right]_{y z} \\
= & \sum_{z \in \Omega_{h}}\left[I-H_{1}-H_{2}\right]_{x z}=0, \quad x \in \Omega_{h}^{\prime} \cup \Omega_{h}^{(2)}, \\
& =1, \quad x \in \Omega_{h}^{(3)} .
\end{aligned}
$$

Now, we consider the characteristic function of $\Omega_{\AA}^{p}$ :

$$
\begin{aligned}
\chi(x) & =1, & & x \in \Omega_{h}^{\prime}, \\
& =0, & & x \in \Omega_{h}^{(2)} \cup \Omega_{h}^{(3)} .
\end{aligned}
$$

Then

$$
\begin{aligned}
& 1 \geqq \chi(x)=\left\{\left[(I-H)^{-1} D\right]\left[D^{-1}(I-H) \chi\right]\right\}_{x} \\
& =\sum_{y \in \Omega_{\boldsymbol{\alpha}}}\left[(I-H)^{-1} D\right]_{x y}\left[D^{-1}(I-H) \chi\right]_{y} \\
& +\sum_{y \in \Omega_{\Delta}(z) \cup \Omega_{h}(s)}\left[(I-H)^{-1} D\right]_{x y}\left[D^{-1}(I-H) \chi\right]_{\nu} \\
& =\sum_{\nu \in \Omega_{\mathbf{\Lambda}}}\left[(I-H)^{-1} D\right]_{x y} \sum_{z \in \Omega_{\Lambda}}\left[D^{-1}(I-H)\right]_{y z} \\
& -\sum_{y \in \Omega_{A^{\prime}}}\left[(I-H)^{-1} D\right]_{x y}\left[D^{-1}(I-H)(1-\chi)\right]_{\nu} \\
& +\sum_{y \in \Omega_{h}(\Omega) \cup \Omega_{A}(s)}\left[(I-H)^{-1} D\right]_{x y}\left[D^{-1}(I-H) \chi\right]_{\nu} \text {. }
\end{aligned}
$$


By (5.4), the first term vanishes. Using the definitions of $H$ and $\chi$, this can be written as

$$
\begin{aligned}
\sum_{y \in \Omega_{h^{\prime}}}\left[(I-H)^{-1} D\right]_{x y} \sum_{y \in \Omega_{A}(2) \cup \Omega_{k}(s)}\left[H_{2}\left(I-H_{1}\right)^{-1} D^{-1}\right]_{y z} \\
-\sum_{y \in \Omega_{h}(2) \cup \Omega_{h}(s)}\left[(I-H)^{-1} D\right]_{x y} \sum_{y \in \Omega_{h},}\left[H_{2}\left(I-H_{1}\right)^{-1} D^{-1}\right]_{y z} \leqq 1 .
\end{aligned}
$$

Now, we estimate the factors in each term. First, note that $(I-H)^{-1} \geqq 0$. This is not obvious, but follows from $H \geqq 0$ and $\rho(H)<1$. That $H \geqq 0$, is due to $0 \leqq$ $H_{2}\left(I-H_{1}\right)^{-1}=H_{2}+H_{2} H_{1}+\cdots$, since the negative terms in $H_{2}$ are cancelled by positive terms in $H_{2} H_{1}$ as in [2]. That $\rho(H)<1$ is due to $\rho(H)=\rho\left(\left(I-H_{1}\right)^{-1} H_{2}\right)<1$, since the row sums of

$$
\begin{aligned}
\left(I-\left(I-H_{1}\right)^{-1} H_{2}\right) & =\left(I-H_{1}\right)^{-1}\left(I-H_{1}-H_{2}\right) \\
& =\left(I-H_{1}-H_{2}\right)+H_{1}\left(I-H_{1}-H_{2}\right)+\cdots
\end{aligned}
$$

are positive. Again negative row sums of $\left(I-H_{1}-H_{2}\right)$ are cancelled by corresponding positive row sums of $H_{1}\left(I-H_{1}-H_{2}\right)$.

Next, for $y \in \Omega_{h}^{(2)} \cup \Omega_{h}^{(3)}$,

$$
\begin{aligned}
\sum_{s \in \Omega_{h^{\prime}}}\left[H_{2}\left(I-H_{1}\right)^{-1}\right. & \left.D^{-1}\right]_{y z} \\
& \leqq \sum_{z \in \Omega_{h}}\left[H_{2}\left(I-H_{1}\right)^{-1} D^{-1}\right]_{y z}=\sum_{z \in \Omega_{h}}\left[D^{-1}-D^{-1}(I-H)\right]_{y z} \\
& \leqq 1-\sum_{z \in \Omega_{h}}\left[D^{-1}(I-H)\right]_{y z}=1-\sum_{z \in \Omega_{h}}\left[I-H_{1}-H_{2}\right]_{y z} \leqq 1 .
\end{aligned}
$$

Now, we consider, for $y \in \Omega_{h}^{\prime \prime}$, the term

$$
\sum_{z \in \Omega_{A}(s) \cup \Omega_{A}(s)}\left[H_{2}\left(I-H_{1}\right)^{-1} D^{-1}\right]_{y z} .
$$

Expanding the summand in a Neumann series, it becomes

$$
\left[\left(H_{2}+H_{2} H_{1}+H_{2} H_{1}^{2}+\cdots\right) D^{-1}\right]_{y s} .
$$

If $y \in \Omega_{h}^{\prime \prime}, z \in \Omega_{h}^{(2)} \cup \Omega_{h}^{(3)}$ is such that $|y-z|=2 h$, then $\left[H_{2}\right]_{y s}=-1 / 60$. However, let $x$ be the point such that $|y-x|=|x-z|=h$. Then $\left[H_{2} H_{1}\right]_{y z}$ contains the term $\left[H_{2}\right]_{y x}\left[H_{1}\right]_{x s}=4 / 225$. Similarly, each negative term in $H_{2} H_{1}^{k}$ is compensated for by a positive term in $H_{2} H_{1}^{k+1}$. Thus, for $y \in \Omega_{h}^{\prime \prime}$,

$$
\sum_{s \in \Omega_{A}(s) \cup \Omega_{A}(s)}\left[H_{2}\left(I-H_{1}\right)^{-1} D^{-1}\right]_{y z} \geqq\left[-\frac{1}{60}+\frac{4}{225}\right] \cdot \frac{1}{2}=\frac{1}{1800} .
$$

It follows from (5.5) and the above that

$$
\sum_{y \in \Omega_{\Omega^{\prime}}}\left[(I-H)^{-1} D\right]_{x y} \leqq 1800\left\{1+\sum_{y \in \Omega_{h}(s) \cup \Omega_{h}(s)}\left[(I-H)^{-1} D\right]_{x y}\right\} .
$$

By similar reasoning, using the function

$$
\begin{array}{rlrl}
\chi(x) & =1, & & x \in \Omega_{h}^{\prime} \cup \Omega_{h}^{(2)}, \\
& =0, & x \in \Omega_{h}^{(3)},
\end{array}
$$

it can be shown that $\sum_{y \in \Omega_{k}(s)}\left[(I-H)^{-1} D\right]_{x y} \leqq C$. The argument is carried out in [2, Lemma 3.3]. Finally, we note from (5.4) that 


$$
1=\sum_{y \in \Omega_{\sharp}}\left[(I-H)^{-1} D\right]_{x y} \sum_{s \in \mathbb{R}_{\sharp}}\left[D^{-1}(I-H)\right]_{y z}=\sum_{y \in \Omega_{\Delta}(s)}\left[(I-H)^{-1} D\right]_{x y} .
$$

Combining the above with (5.7), we see that

$$
\sum_{\nu \in \Omega_{\aleph^{\prime}}}\left[(I-H)^{-1} D\right]_{x y} \leqq C .
$$

From (5.2) and (5.3), we finally have

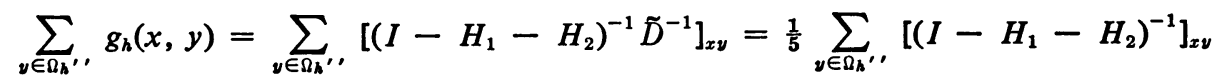

$$
\begin{aligned}
& =\frac{1}{5} \sum_{y \in \Omega_{h^{\prime \prime}}} \sum_{s \in \Omega_{A^{\prime}} \cup \operatorname{QRh}(s)}\left\{\left[D\left(I-H_{1}\right)\right]^{-1}\right\}_{x z}\left[(I-H)^{-1} D\right]_{s y} \\
& \leqq \frac{1}{5} \max _{z \in \Omega_{B^{\prime}} \cup \Omega_{h}(z)} \sum_{v \in \mathbb{R}_{h^{\prime}}}\left[(I-H)^{-1} D\right]_{z y} \text {, }
\end{aligned}
$$

or, from (5.9),

$$
\sum_{\nu \in \Omega_{h^{\prime}}} g_{h}(x, y) \leqq C,
$$

the desired estimate.

We next define another Green's function $G_{h}$ by

$$
-\Delta_{h} G_{h}(x, y)=h^{-2} \delta(x, y), \quad x, y \in \Omega_{h} .
$$

Although $G_{h}$ may not be nonnegative, it is a perturbation of $g_{h}$. We have THEOREM 5.1. For any mesh function $S$,

$$
\begin{aligned}
& \max _{x \in \Omega_{h}} \sum_{y \in \Omega_{h}}\left|\left[G_{h}(x, y)-g_{h}(x, y)\right] S(y)\right| \\
& \leqq C\left[\max _{\Omega_{h}(z)}|S|+\max _{x \in \Omega_{A^{\prime}}, \cup \Omega_{h}(s) \cup \Omega_{A}(s)} \sum_{y \in \Omega_{h}} g_{h}(x, y)|S(y)|\right] .
\end{aligned}
$$

Proof. Let $x_{0} \in \Omega$ be the point where $\sum_{y \in \mathrm{a}_{h}}\left|\left[G_{h}(x, y)-g_{h}(x, y)\right] S(y)\right|$ attains its maximum and let

$$
W(x)=\sum_{y \in \Omega_{h}}\left[G_{h}(x, y)-g_{h}(x, y)\right] S^{*}(y),
$$

where $S^{*}(y)=|S(y)| \operatorname{sgn}\left[G_{h}\left(x_{0}, y\right)-g_{h}\left(x_{0}, y\right)\right]$. Employing (4.9), we have

$$
\begin{aligned}
\max _{\Omega_{h}}|W| & \leqq C \max _{\Omega_{h}(s)}\left|h^{2} \Delta_{h} W\right| \\
& \leqq C\left[\max _{\Omega_{h}(s)}|S|+\max _{x \in \Omega_{h^{\prime}}, \Omega_{\Omega_{A}(s) \cup \Omega_{h}(s)}}\left|\sum g_{h}(x, y) S^{*}(y)\right|\right] .
\end{aligned}
$$

and (5.12) follows.

COROLLARY 5.2. For all $x, z \in \Omega_{h}$,

$$
\begin{aligned}
\sum_{y \in \Omega_{\Omega^{\prime}} \cup \cup \Omega_{h}\left(x^{2}\right) \cup \Omega_{h}(s)}\left|G_{h}(x, y)\right| & \leqq C, \\
h^{2} \sum_{y \in \Omega_{h}}\left|G_{h}(x, y)\right| & \leqq C, \\
\left|G_{h}(x, z)\right| & \leqq C|\log h|, \\
h^{2} \sum_{\nu \in \Omega_{h}}\left|G_{h}(x, y)\right|^{2} & \leqq C,
\end{aligned}
$$


and for $|x-\partial \Omega| \leqq C h$,

$$
h^{2} \sum_{y \in \Omega_{k}}\left|G_{h}(x, y)\right| \leqq C h .
$$

Proof. For (5.13), employ the characteristic function of $\Omega_{h}^{\prime \prime} \cup \Omega_{h}^{(2)} \cup \Omega_{h}^{(3)}$ as $S$ in (5.13). Then apply the triangle inequality and (4.4), (4.5), and (5.10). For (5.14), let $S=h^{2}$ and use (4.6) and (4.10), respectively. For (5.15), let $S(y)=\delta(y, z)$ in (5.12), apply the triangle inequality and (4.11). For (5.16), let $x_{0}$ be the point where $\max _{x \in \Omega_{h}} h^{2} \sum_{y \in \Omega_{h}}\left|G_{h}(x, y)\right|^{2}$ is attained, and let $S(y)=h^{2} G_{h}\left(x_{0}, y\right)$ in (5.12), from which it follows that

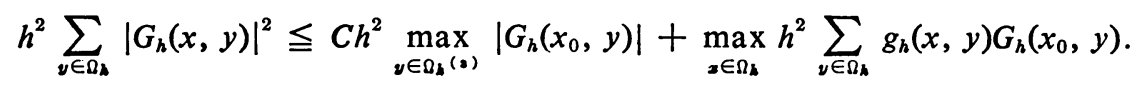

Again, using (5.12) with $S(y)=h^{2} g_{h}(x, y)$ for $x$ fixed,

$$
h^{2} \sum_{\nu \in \Omega_{\Lambda}} G_{h}\left(x_{0}, y\right) g_{h}(x, y) \leqq C h^{2} \max _{y \in \Omega_{\Lambda}(s)}\left|g_{h}(x, y)\right|+\max _{x_{\bullet} \in \Omega_{\Lambda}} h^{2} \sum_{\nu \in \Omega_{\Lambda}} g_{h}\left(x_{0}, y\right) g_{h}(x, y) .
$$

By (4.11), this term can be seen to be bounded. Finally, letting $S(y)=h^{2} \delta\left(y_{0}, y\right)$ in (5.12), we have, for any $y_{0} \in \Omega_{h}$,

$$
\left|h^{2} G_{h}\left(x_{0}, y_{0}\right)\right| \leqq C\left[h^{2}+\max _{x \in \Omega_{\Lambda}} h^{2} g_{h}\left(x, y_{0}\right)\right],
$$

which indeed tends to zero as $h$ does, by (4.11), and (5.16) follows. For (5.17) use $S=h^{2}$ and (4.10).

We require yet one more Green's function $G_{h}^{\prime}$ defined by

$$
\text { (5.18) }-\Delta_{h} G_{h}^{\prime}(x, y)=h^{-2} \delta(x, y), \quad x \in \Omega_{h}^{\prime}, \quad G_{h}^{\prime}(x, y)=0, x \in \Omega_{h}^{(2)} \cup \Omega_{h}^{(3)} \text {, }
$$

for all $y \in \Omega_{\mathrm{k}}$. Thus, the matrix $\left[G_{h}^{\prime}(x, y)\right]_{x, y \in \Omega_{\alpha}}$ is the inverse of the symmetric matrix

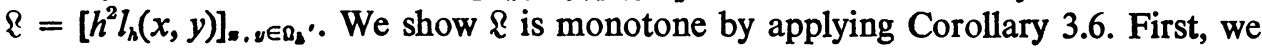
show $\mathbb{R}+\frac{1}{3} I$ monotone from Corollary 3.5: we define $M_{1}$ by

$$
\begin{aligned}
{\left[M_{1}\right]_{x y} } & =\frac{16}{3}, & & x=y, \\
& =-\frac{4}{3}, & & |x-y|=h, \\
& =0, & & \text { otherwise, }
\end{aligned}
$$

for $x, y \in \Omega_{k}^{\prime}$, and we define

$$
\begin{aligned}
{\left[\boldsymbol{M}_{2}\right]_{\mathbf{x}} } & =\frac{8}{\sqrt{12}}, & & x=y, \\
& =-\frac{1}{112}, & & |x-y|=k \\
& =0, & & \text { otherwise }
\end{aligned}
$$

Since $M_{:}$and $M_{\varepsilon}$ are of positive type, they are monotone, hence, so is $M_{2,}^{z}$ and it is cisiy to see that

$$
\text { M. } \leqq \& \div \frac{1}{2} I \leq M^{2}
$$


Thus, $\mathfrak{R}$ is monotone if its eigenvalues, necessarily real by symmetry, are positive. But these are $h^{2} \mu_{k}^{(i)}$, where $\mu_{h}^{(i)}$ is the $i$ th eigenvalue satisfying

$$
\Delta_{h} V_{h}^{(i)}(x)+\mu_{h}^{(i)} V_{h}^{(i)}(x)=0, x \in \Omega_{h}^{\prime}, \quad V_{h}^{(i)}(x)=0, x \in \Omega_{h}^{(2)} \cup \Omega_{h}^{(3)} .
$$

In the next section, we shall show that indeed $\left|\mu_{h}^{(i)}-\lambda^{(i)}\right| \rightarrow 0$ as $h \rightarrow 0$, for $\lambda^{(i)}$ the $i$ th eigenvalue of (1.1), which is strictly positive. Thus, for $h$ sufficiently small, $\mathbb{R}$ is monotone and $G_{h}^{\prime}$ nonnegative. Thus, as a consequence of Lemma 3.7,

$$
0 \leqq G_{h}^{\prime}(x, y) \leqq g_{h}(x, y), \quad x, y \in \Omega_{h} .
$$

From (5.20), we see that all of the inequalities proved for $g_{h}$ hold for $G_{h}^{\prime}$. In particular, the difficult inequality (5.10) does, from which we prove the key inequality

$$
\max _{\Omega_{B}}|W| \leqq C\left[\max _{\Omega_{\mathbf{B}^{\prime}}}\left|\Delta_{h} W\right|+\max _{\Omega_{h}(s) \cup \Omega_{B^{\prime}}(s)}|W|\right],
$$

for all $W$ defined on $\Omega_{h}$. To prove this, let

$$
\begin{aligned}
W^{*}(x) & =W(x), & & x \in \Omega_{h}^{\prime}, \\
& =0, & & x \in \Omega_{h}^{(2)} \cup \Omega_{h}^{(3)} .
\end{aligned}
$$

Then, by (5.18),

$$
\begin{aligned}
W^{*}(x) & =h^{2} \sum_{\nu \in \Omega_{\boldsymbol{b}^{\prime}},} G_{h}^{\prime}(x, y)\left[-\Delta_{h} W^{*}(y)\right] \\
& =h^{2} \sum_{\nu \in \Omega_{\mathbf{\Omega}^{\prime}}} G_{h}^{\prime}(x, y)\left[-\Delta_{h} W(y)\right]+h^{2} \sum_{\nu \in \Omega_{\mathbf{k}^{\prime}}} G_{h}^{\prime}(x, y)\left[\Delta_{h} W(y)-\Delta_{h} W^{*}(y)\right],
\end{aligned}
$$

and (5.21) follows from (4.6), (5.10), and (5.20).

6. Convergence of $\mu_{h}^{(n)}$ to $\lambda^{(n)}$. In this section, we show that the eigenvalue $\mu_{\hbar}^{(n)}$ of

(6.1) $\Delta_{h} V_{h}^{(n)}(x)+\mu_{h}^{(n)} V_{h}^{(n)}(x)=0, \quad x \in \Omega_{h}^{\prime}, \quad V_{h}^{(n)}(x)=0, \quad x \in \Omega_{h}^{(2)} \cup \Omega_{h}^{(3)}$, converges to $\lambda^{(n)}$ of (1.1) for each $n$. We will use the variational principles associated with (1.1) and (6.1), and a technique of Weinberger [9].

The $n$th eigenvalue of (1.1) can be characterized by

$$
\lambda^{(n)}=\min \max D(u) / \int_{\varepsilon} u^{2} d x,
$$

where $u=\alpha_{1} u_{1}+\cdots+\alpha_{n} u_{n}$, the max is with respect to the scalars $\alpha_{1}, \cdots, \alpha_{n}$, the min is with respect to choices of linearly independent $u_{1}, \cdots, u_{n}$, continuous, piecewise differentiable functions vanishing on $\partial \Omega$, and $D(u)$ is the Dirichlet integral.

Similarly, the $n$th eigenvalue of $(6.1)$ can be characterized by

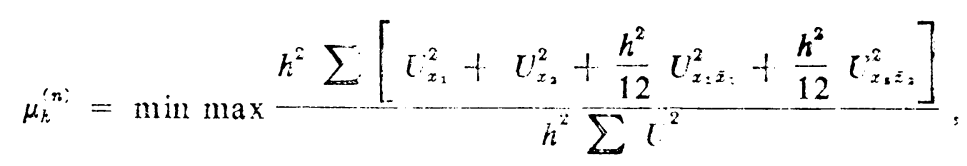

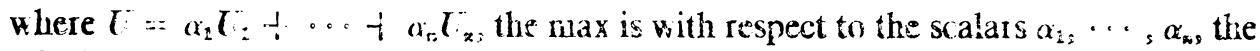
min is with respect to choices of linearly independent mesh functions $\boldsymbol{C}_{1}, \ldots{ }_{s} \boldsymbol{U}_{\infty}$ vauishing on $\Omega_{k}^{(2)} \Omega_{\alpha}^{(3)}$, the sum is erer the mesh points of $\Omega_{i}^{i}$ and subscript $x_{i}\left(\bar{x}_{i}\right)$ 
denotes forward (backward) difference quotient in the $x_{i}$ direction, $i=1,2$, i.e., $U_{x_{1}}\left(y_{1}, y_{2}\right)=\left[U\left(y_{1}+h, y_{2}\right)-U\left(y_{1}, y_{2}\right)\right] / h$, etc.

First, we show

$$
\mu_{h}^{(n)} \leqq \lambda^{(n)}+O(h) .
$$

Let $u^{(1)}, \cdots, u^{(n)}$ be eigenfunctions associated with $\lambda^{(1)}, \cdots, \lambda^{(n)}$ in (1.1), $u=\alpha_{1} u^{(1)}+\cdots+\alpha_{n} u^{(n)}$, and define

$$
\begin{aligned}
u(x) & =h^{-1} \int_{O_{\mathbf{b}}(x)} u(y) d y, & & x \in \Omega_{h}^{\prime}, \\
& =0, & & x \in \Omega_{h}^{(2)} \cup \Omega_{h}^{(3)},
\end{aligned}
$$

where $Q_{h}(x)=\left\{\left(y_{1}, y_{2}\right):\left|x_{1}-y_{1}\right| \leqq \frac{1}{2} h,\left|x_{2}-y_{2}\right| \leqq \frac{1}{2} h\right\}$ is the square of side $h$ centered at $x$. Put this $U$ in (6.3). Employing inequalities (2.14), (2.22) and (8.6) of Weinberger [9], we see that

$$
\mu_{h}^{(n)} \leqq \max _{\alpha} \frac{D(u)+\frac{h^{2}}{12} \int_{\Omega}\left\{\left(\frac{\partial^{2} u}{\partial x_{1}^{2}}\right)^{2}+\left(\frac{\partial^{2} u}{\partial x_{2}^{2}}\right)^{2}\right\} d x}{\int_{\Omega} u^{2} d x-\left(h^{2} / \pi^{2}\right) D(u)},
$$

and Hubbard [5, pp. 568-569], has shown

$$
\int_{\Omega}\left\{\left(\frac{\partial^{2} u}{\partial x_{1}^{2}}\right)^{2}+\left(\frac{\partial^{2} u}{\partial x_{2}^{2}}\right)^{2}\right\} d x \leqq C\left(\lambda^{(n)}\right)^{2} .
$$

From these, (6.4) follows.

Next, we show

$$
\lambda^{(n)} \leqq \mu_{h}^{(n)}+O(h) .
$$

Let $V_{h}^{(1)}, \cdots, V_{h}^{(n)}$ be eigenvectors associated with $\mu_{h}^{(1)}, \cdots, \mu_{b}^{(n)}$ in (6.1), $U=\alpha_{1} V_{h}^{(1)}+\cdots+\alpha_{n} V_{h}^{(n)}$, and define $u$ to be the continuous, piecewise linear function interpolating $U$ (see [9, Section 6]). Then, by (6.4), (6.7) of [9] we see that

$$
\begin{aligned}
& \lambda^{(n)} \leqq \max _{\alpha} \frac{h^{2} \sum\left(U_{x_{1}}^{2}+U_{x_{2}}^{2}\right)}{h^{2} \sum U^{2}-\frac{1}{4} h^{4} \sum\left(U_{x_{1}}^{2}+U_{x_{s}}^{2}\right)}
\end{aligned}
$$

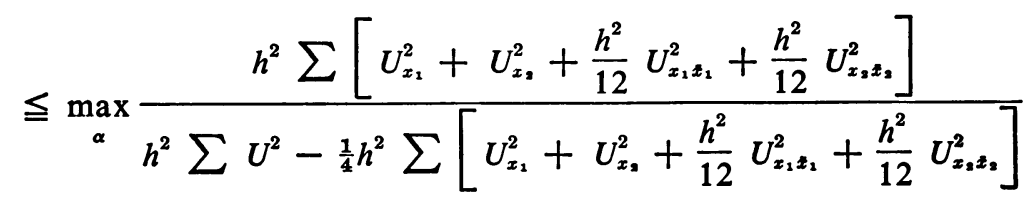

$$
\begin{aligned}
& =\frac{\mu_{h}^{(n)}}{1-\frac{1}{4} h^{2} \mu_{h}^{(n)}}
\end{aligned}
$$

and we obtain (6.5). Combining (6.4) and (6.5), we have

$$
\left|\mu_{h}^{(n)}-\lambda^{(n)}\right| \rightarrow 0 \quad \text { as } h \rightarrow 0,
$$

for each $n=1,2, \cdots$. 
7. Convergence of $\lambda_{h}^{(n)}$ to $\lambda^{(n)}$ by Perturbation. Next, we will show that the $\lambda_{h}^{(n)}$ are a perturbation of the $\mu_{h}^{(n)}$, and that as $h$ tends to zero, $\lambda_{h}^{(n)}$ tends to $\mu_{h}^{(n)}$, hence to $\lambda^{(n)}$, by Section 6 . We employ the following theorem of Wielandt:

THEOREM 7.1. If $A, B$ are $\nu \times \nu$ matrices and $A$ has an orthonormal basis of eigenvectors, then the eigenvalues of $B$ lie in the union of the $\nu$ discs $\left|\mu^{(i)}-z\right| \leqq\|A-B\|_{2}$, where the $\mu^{(i)}$ are the eigenvalues of $A$. If $k$ discs are disjoint from the others, they contain exactly $k$ eigenvalues of $B$.

In the theorem, $\|\cdot\|_{2}$ is the spectral norm of a matrix, defined by

$$
\|M\|_{2}=\sup _{\xi}\|M \xi\|_{2} /\|\xi\|_{2} \text {, where }\|\xi\|_{2}=\left(\sum_{i=1}^{j}\left|\xi_{i}\right|^{2}\right)^{1 / 2}
$$

for a $\nu$-vector $\xi=\left(\xi_{1}, \cdots, \xi_{v}\right)$. For a proof of the theorem, see [6].

We apply the theorem as follows. For $A$, we take the matrix $\left[h^{2} G_{h}^{\prime}(x, y)\right]_{x, y \in \mathbf{a}_{h}}$. Note that the minor $\left[h^{2} G_{h}^{\prime}(x, y)\right]_{x, y \in \Omega_{a}}$ is symmetric, while $h^{2} G_{h}^{\prime}(x, y)=0$ for $x \in$ $\Omega_{h}^{(2)} \cup \Omega_{h}^{(3)}$, so that $A$ has an orthonormal basis of eigenvectors, and the eigenvalues are simply $\left[\mu_{h}^{(i)}\right]^{-1}$ plus some zeros. For $B$, we take the matrix $\left[h^{2} G_{h}(x, y)\right]$ whose eigenvalues are $\left[\lambda_{h}^{(i)}\right]^{-1}$. Thus, we must estimate $\left\|h^{2}\left(G_{h}-G_{h}^{\prime}\right)\right\|_{2}$. However, for any matrix,

$$
\|M\|_{2} \leqq\left[\rho\left(M M^{T}\right)\right]^{1 / 2} \leqq\left\|M M^{T}\right\|_{1}^{1 / 2},
$$

where $\|\cdot\|_{1}$ is the maximum of the absolute row sums of the matrix. This is a consequence of the Gerschgorin circle theorem (see, e.g., [7, p. 146]). Thus, we need to estimate

$$
h^{4} \max _{x \in \Omega_{h}} \sum_{y \in \Omega_{h}}\left|\sum_{z \in \Omega_{h}}\left[G_{h}(x, z)-G_{h}^{\prime}(x, z)\right]\left[G_{h}(y, z)-G_{h}^{\prime}(y, z)\right]\right| .
$$

Let $x_{0}$ be the point where the max is attained and put

$$
\sigma(y)=\operatorname{sgn} \sum_{s \in \Omega_{h}}\left[G_{h}\left(x_{0}, z\right)-G_{h}^{\prime}\left(x_{0}, z\right)\right]\left[G_{h}(y, z)-G_{h}^{\prime}(y, z)_{\rfloor} .\right.
$$

Then, let

$$
W(x)=h^{4} \sum_{y, z \in \Omega_{h}}\left[G_{h}(x, z)-G_{h}^{\prime}(x, z)\right]\left[G_{h}(y, z)-G_{h}^{\prime}(y, z)\right] \sigma(y)
$$

in (4.9). Then, (7.1) is bounded by

$$
\begin{aligned}
C h^{4} \max _{z \in \Omega_{A}(s)} \sum_{y \in \Omega_{\Lambda}} \mid G_{h}(y, z) & -G_{h}^{\prime}(y, z) \mid \\
& +C h^{4} \max _{x \in \Omega_{\Lambda^{\prime}}} \sum_{z \in \Omega_{h^{\prime}}} G_{h}^{\prime}(x, z) \sum_{y \in \Omega_{\Lambda}}\left|G_{h}(y, z)-G_{h}^{\prime}(y, z)\right| .
\end{aligned}
$$

Now,

$$
h^{2} \sum_{\nu \in \Omega_{\Lambda}}\left|G_{h}(y, z)-G_{h}^{\prime}(y, z)\right| \leqq C \max _{\nu, z \in \Omega_{h}}\left[\left|G_{h}(y, z)\right|+G_{h}^{\prime}(y, z)\right] \leqq C|\log h|,
$$

by (4.11), (5.15) and (5.20). Using this in (7.2) and also (4.10) and (5.20), we have (7.2) bounded by $C h|\log h|$, which tends to zero as $h$ tends to zero. Thus, the radii of the discs in Theorem 7.1 tend to zero as $h$ does. Since the $\mu_{h}^{(n)}$ tend to the $\lambda^{(n)}$, which have no finite accumulation point, the disc associated with $\left[\mu_{h}^{(n)}\right]^{-1}$ for any 
fixed $n$ eventually becomes disjoint from the remaining discs. Consequently, for any fixed $n$ and $\epsilon>0$, there is $h$ sufficiently small that

$$
\left|\lambda_{h}^{(n)}-\lambda^{(n)}\right|<\epsilon .
$$

8. Main Theorem. We are now ready to state and prove our main theorem:

THEOREM 8.1. Let $\lambda^{(n)}$ be the nth eigenvalue of (1.1), let $\lambda_{h}^{(n)}$ be the nth eigenvalue of (2.6) with associated eigenvector $U_{h}^{(n)}$. For each $n=1,2, \cdots$, there are constants $C_{n}, h_{n}$ such that for $h<h_{n}$

$$
\left|\lambda_{h}^{(n)}-\lambda^{(n)}\right|<C_{n} h^{4},
$$

and there is an eigenfunction $u^{(n)}$ associated with $\lambda^{(n)}$ such that

$$
\max _{\Omega_{A}}\left|U_{h}^{(n)}-u^{(n)}\right|<C_{n} h^{4}
$$

Proof. With the machinery generated in the previous sections, our proof will have exactly the form of the proof of the corresponding Theorem 5.1 of [6]. For this reason, we only sketch the proof.

By (7.3)

$$
\left|\lambda_{h}^{(n)}\right| \leqq C_{n}
$$

By (5.11), (2.6) is equivalent to

$$
U_{h}^{(n)}(x)=\lambda_{h}^{(n)} h^{2} \sum_{y \in \Omega_{h}} G_{h}(x, y) U_{h}^{(n)}(y), \quad x \in \Omega_{h} .
$$

Let us use the notations

$$
\begin{aligned}
& \langle U, V\rangle_{\boldsymbol{h}} \equiv h^{2} \sum_{y \in \Omega_{\boldsymbol{\Lambda}}} U(y) \overline{V(y)}, \quad\|U\|_{h} \equiv\langle U, U\rangle_{h}^{1 / 2}, \\
& \langle U, V\rangle_{h}^{\prime} \equiv h^{2} \sum_{\nu \in \Omega_{h}} U(y) \overline{V(y)}, \quad\|U\|_{h}^{\prime} \equiv\langle U, U\rangle_{h}^{\gamma^{1 / 2}} .
\end{aligned}
$$

If $U_{h}^{(n)}$ is normalized by requiring $\left\|U_{\Lambda}^{(n)}\right\|_{h}=1$, then (8.4), (8.3), the Schwarz inequality, and (5.16) show

$$
\max _{\mathbf{n}_{\mathrm{A}}}\left|U_{h}^{(n)}\right| \leqq C_{n} .
$$

From (8.4), (8.5) and (5.17), we see that for $|x-\partial \Omega| \leqq C h$

$$
\left|U_{h}^{(n)}(x)\right| \leqq C_{n} h .
$$

Let us suppose that $\lambda^{(n)}=\lambda^{(n+1)}=\cdots=\lambda^{(n+m)}$ is an eigenvalue of multiplicity $m+1$. Since $\Delta_{h}$ restricted to $\Omega_{h}^{\prime}$ is symmetric, the eigenvectors $V_{h}^{(n)}$ of (6.1) are a complete orthonormal basis on $\Omega_{h}^{\prime}$ :

$$
\left\langle V_{h}^{(i)}, V_{h}^{(i)}\right\rangle_{h}^{\prime}=\delta(i, j) .
$$

If we set

$$
\tilde{V}_{h}^{(i)}=\sum_{i=n}^{n+m}\left\langle U_{h}^{(i)}, V_{h}^{(i)}\right\rangle_{h}^{\prime} V_{h}^{(i)}, \quad i=n, \cdots, n+m,
$$

then 


$$
\left\|U_{h}^{(i)}-\tilde{V}_{h}^{(i)}\right\|_{h}^{\prime} \leqq C_{n} h, \quad i=n, \cdots, n+m .
$$

This follows from Parseval's identity:

$$
\begin{aligned}
&\left\|U_{h}^{(i)}\right\|_{h}^{2}=\left\langle U_{h}^{(i)}, \tilde{V}_{h}^{(i)}\right\rangle_{h}^{\prime}+\sum_{i \neq n}, \cdots, n+m \\
&\left.=\left\langle U_{h}^{(i)}, \tilde{V}_{h}^{(i)}\right\rangle_{h}^{\prime}+\sum_{i \neq n}^{(i)}, V_{h}^{(i)}\right\rangle\left._{h}^{\prime}\right|^{2} \\
&\left|\frac{\mu_{h}^{(i)}}{\mu_{h}^{(i)}-\lambda_{h}^{(i)}}\left\langle H_{h}^{(i)}, V_{h}^{(i)}\right\rangle_{h}^{\prime}\right|^{2},
\end{aligned}
$$

where $H_{\Lambda}^{(i)}$ is uniquely defined by

$$
\Delta_{h} H_{h}^{(i)}(x)=0, \quad x \in \Omega_{h}^{\prime}, \quad H_{h}^{(i)}(x)=U_{h}^{(i)}(x), \quad x \in \Omega_{h}^{(2)} \cup \Omega_{h}^{(3)} .
$$

It follows from our hard-won inequality (5.21) that

$$
\max _{\Omega_{A}}\left|H_{h}^{(i)}\right| \leqq \max _{\Omega_{h}^{(2)} \cup \Omega_{A}^{(s)}}\left|U_{h}^{(i)}\right| \leqq C_{i} h,
$$

by (8.6), and so

$$
\left\|U_{h}^{(i)}-\tilde{V}_{h}^{(i)}\right\|_{h}^{2} \equiv\left\|U_{h}^{(i)}\right\|_{h}^{2}-\left\langle U_{h}^{(i)}, \tilde{V}_{h}^{(i)}\right\rangle_{h}^{\prime} \leqq C_{i} h^{2} .
$$

In a very similar manner, we show that if

$$
\tilde{V}_{h}^{(i)}=\sum_{i=n}^{n+m}\left\langle U^{(i)}, V_{h}^{(i)}\right\rangle_{h}^{\prime} V_{h}^{(i)}, \quad i=n, \cdots, n+m,
$$

then

$$
\left\|u^{(i)}-\tilde{V}_{h}^{(i)}\right\|_{h}^{\prime} \leqq C_{n} h, \quad i=n, \cdots, n+m .
$$

From (8.8), we can conclude that the $(m+1) \times(m+1)$ matrix $\left[\left\langle u^{(i)}, V_{\Lambda}^{(i)}\right\rangle_{h}^{\prime}\right]$, $i, j=n, \cdots, n+m$, is nonsingular. In particular then, there are eigenvectors

$$
u_{h}^{(i)}=\sum_{i=n}^{n+m} a_{i i}(h) u^{(i)}, \quad i=n, \cdots, n+m,
$$

in the eigenmanifold associated with $\lambda^{(n)}$ such that

$$
\left\langle u_{h}^{(i)}, V_{h}^{(i)}\right\rangle_{h}^{\prime}=\left\langle U_{h}^{(i)}, V_{h}^{(i)}\right\rangle_{h}^{\prime}, \quad i, j=n, \cdots, n+m .
$$

Moreover, the coefficients $a_{i j}(h)$ are bounded independently of $h$.

Then, it follows from (8.9) and Parseval's identity that

$$
\begin{aligned}
\left\|U_{h}^{(i)}-u_{h}^{(i)}\right\|_{h}^{2}= & h^{2} \sum_{\Omega_{A}(2) \cup \Omega_{h}^{(3)}}\left|U_{h}^{(i)}-u_{h}^{(i)}\right|^{2}+\sum_{i \neq n, \cdots, n+m}\left|\left\langle U_{h}^{(i)}-u_{h}^{(i)}, V_{h}^{(i)}\right\rangle_{h}^{\prime}\right|^{2} \\
= & h^{2} \sum_{\Omega_{h}(2) \cup \Omega_{h}(i)}\left|U_{h}^{(i)}-u_{h}^{(i)}\right|^{2} \\
& +\sum_{i \neq n, \cdots, n+m}\left|\frac{\mu_{h}^{(i)}}{\mu_{h}^{(i)}-\lambda_{h}^{(i)}}\left\langle H_{h}^{(i)}, V_{h}^{(i)}\right\rangle_{h}^{\prime}-\frac{\mu_{h}^{(j)}}{\mu_{h}^{(i)}-\lambda^{(i)}}\left\langle\tilde{H}_{h}^{(i)}, V_{i}^{(i)}\right\rangle_{h}^{\prime}\right|^{2},
\end{aligned}
$$

where $\tilde{H}_{a}^{(i)}$ is defined by

$$
\Delta_{\lambda} \widetilde{H}_{h}^{(i)}(x)=0, \quad x \in \Omega_{h}^{\prime}, \quad \tilde{H}_{h}^{(i)}(x)=u_{h}^{(i)}(x), \quad x \in \Omega_{h}^{(2)} \cup \Omega_{h}^{(3)} .
$$

Since $\left|u_{h}^{(i)}(x)\right| \leqq C_{i} h$ for $|x-\partial \Omega| \leqq C h$, we see that

$$
\left\|U_{h}^{(i)}-u_{h}^{(i)}\right\|_{h} \leqq C_{i} h .
$$


From (8.10), we also have

$$
\left|\left\langle U_{h}^{(i)}, u_{h}^{(i)}\right\rangle_{h}\right| \geqq 1-C_{i} h^{2} .
$$

Inequality (8.11) is the key inequality needed to prove the first half of Theorem 8.1, for now

$$
\begin{aligned}
\left.\lambda_{h}^{(i)}-\lambda^{(i)}\right)\left\langle U_{h}^{(i)}, u_{h}^{(i)}\right\rangle= & \left\langle U_{h}^{(i)}, \Delta u_{h}^{(i)}-\Delta_{h}^{*} u_{h}^{(i)}\right\rangle_{h} \\
= & \left\langle U_{h}^{(i)}, \tau_{h} u_{h}^{(i)}\right\rangle_{h}-\left\langle u_{h}^{(i)}, \tau_{h} u_{h}^{(i)}\right\rangle_{h}+\left\langle\tau_{h} u_{h}^{(i)}, u_{h}^{(i)}\right\rangle_{h} \\
& +\left\langle U_{h}^{(i)}-u_{h}^{(i)}, \Delta_{h} u_{h}^{(i)}-\Delta_{h}^{*} u_{h}^{(i)}\right\rangle_{h},
\end{aligned}
$$

obtained by adding and subtracting terms. We have used the notations

$$
\tau_{h} u_{h}^{(i)} \equiv \Delta u_{h}^{(i)}-\Delta_{h} u_{h}^{(i)}
$$

for the truncation error, and $\Delta_{h}^{*}$ for the adjoint of $\Delta_{h}$ defined by

$$
\Delta_{h}^{*} V(x)=\sum_{y \in \Omega_{h}} l_{h}(y, x) V(y) .
$$

Recall by (2.6) and our smoothness assumption on $u^{(i)}$ that

$$
\begin{aligned}
\left|\tau_{h} u_{h}^{(i)}\right| & \leqq C_{i} h^{4}, & & \text { on } \Omega_{h}^{\prime}, \\
& \leqq C_{i} h^{2}, & & \text { on } \Omega_{h}^{(2)} \cup \Omega_{h}^{(3)} .
\end{aligned}
$$

However, on $\Omega_{h}^{(2)} \cup \Omega_{h}^{(3)}$ both $U_{h}^{(i)}$ and $u_{h}^{(i)}$ are bounded by $C_{i} h$, while the number of points in $\Omega_{h}^{(2)} \cup \Omega_{h}^{(3)}$ is only proportional to $h^{-1}$. From these considerations, we see that the first three terms on the right side of (8.12) are bounded by $C_{i} h^{4}$. As for the remaining term,

$$
\Delta_{h} u_{h}^{(i)}(x)-\Delta_{h}^{*} u_{h}^{(i)}(x)
$$

vanishes for $x \notin \Omega_{h}^{\prime \prime} \cup \Omega_{h}^{(2)} \cup \Omega_{h}^{(3)}$, and is bounded by

$$
C h^{-2} \max _{\Omega_{B^{\prime}}, \cup \Omega_{h}(s) \cup \Omega_{B}(s)}\left|u_{h}^{(i)}\right| \leqq C_{i} h^{-1}
$$

for $x \in \Omega_{h}^{\prime \prime} \cup \Omega_{h}^{(2)} \cup \Omega_{h}^{(3)}$. Again noting that the number of points in $\Omega_{h}^{\prime \prime} \cup \Omega_{h}^{(2)} \cup \Omega_{h}^{(3)}$ is only proportional to $h^{-1}$, the last term on the right of (8.12) is bounded by

$$
C_{i} \max _{\Omega_{A}, \Omega_{\Omega_{A}(s) \cup \Omega_{h}(s)}}\left|U_{h}^{(i)}-u_{h}^{(i)}\right| \text {. }
$$

Thus, using (8.11) we have the inequality

$$
\left|\lambda_{h}^{(i)}-\lambda^{(i)}\right| \leqq C_{i}\left[\max _{\Omega_{h^{\prime}} \cup \Omega_{h}(z) \cup \Omega_{h}(s)}\left|U_{h}^{(i)}-u_{h}^{(i)}\right|+h^{4}\right] .
$$

We next employ the discrete Green's function to write

$$
\begin{aligned}
U_{h}^{(i)}(x)-u_{h}^{(i)}(x)= & h^{2} \sum_{\nu \in \Omega_{h}} G_{h}(x, y) \Delta_{h}\left[u_{h}^{(i)}(y)-U_{h}^{(i)}(y)\right] \\
= & -h^{2} \sum_{y \in \Omega_{h}} G_{h}(x, y) \tau_{h} u_{h}^{(i)}(y)+\lambda^{(i)} h^{2} \sum_{y \in \Omega_{h}} G_{h}(x, y)\left[U_{h}^{(i)}(y)-u_{h}^{(i)}(y)\right] \\
& +\left(\lambda_{h}^{(i)}-\lambda^{(i)}\right) h^{2} \sum_{y \in \Omega_{h}} G_{h}(x, y) U_{h}^{(i)}(y) .
\end{aligned}
$$

Using inequalities (5.13) and (5.14), we see that the first term on the right of (8.14) is bounded by $C_{i} h^{4}$. By (5.14) and (8.5) the last term on the right is bounded by 
$C_{i}\left|\lambda_{h}^{(i)}-\lambda^{(i)}\right|$, or if $|x-\partial \Omega| \leqq C h,(5.17)$ shows the last term bounded by $C_{i} h$ $\left|\lambda_{h}^{(i)}-\lambda^{(i)}\right|$. Using (8.3), (5.16) and Schwarz's inequality bound the middle term on the right by $\left\|U_{h}^{(i)}-u_{h}^{(i)}\right\|_{h}$, or, if $|x-\partial \Omega| \leqq C h$, (5.17) bounds it by $C_{i} h \max _{\mathrm{a}_{\mathrm{A}}}\left|U_{h}^{(i)}-u_{h}^{(i)}\right|$. In summary,

$$
\begin{aligned}
& \max _{\Omega_{A}}\left|U_{h}^{(i)}-u_{h}^{(i)}\right| \leqq C_{i}\left[\left\|U_{h}^{(i)}-u_{h}^{(i)}\right\|_{h}+\left|\lambda_{h}^{(i)}-\lambda^{(i)}\right|+h^{4}\right],
\end{aligned}
$$

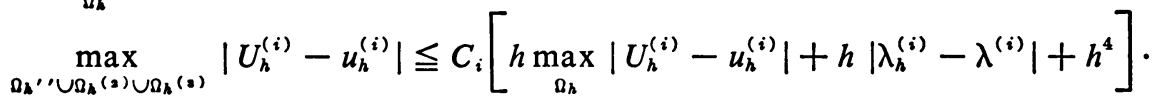

Finally, we use Parseval's identity and (8.9) to conclude that

$$
\left\|U_{h}^{(i)}-u_{h}^{(i)}\right\|_{h}^{2}=h^{2} \sum_{\Omega_{A}\left(s, \cup \Omega_{h}(0)\right.}\left|U_{h}^{(i)}-u_{h}^{(i)}\right|^{2}+\sum_{i \ngtr n} \sum_{\cdots, n+m}\left|\left\langle U_{h}^{(i)}-u_{h}^{(i)}, V_{h}^{(i)}\right\rangle_{h}^{\prime}\right|^{2},
$$

and by a straightforward computation

$$
\left(\mu_{h}^{(i)}-\lambda^{(i)}\right)\left\langle U_{h}^{(i)}-u^{(i)}, V_{h}^{(i)}\right\rangle_{h}^{\prime}=\left\langle\left(\lambda_{h}^{(i)}-\lambda^{(i)}\right) U_{h}^{(i)}-\tau_{h} u_{h}^{(i)}+\widetilde{H}_{h}^{(i)}, V_{h}^{(i)}\right\rangle_{h}^{\prime},
$$

where $\widetilde{\tilde{H}}_{h}^{(i)}$ is defined by

$$
\Delta_{h} \widetilde{H}_{h}^{(i)}(x)=0, \quad x \in \Omega_{h}^{\prime}, \quad \widetilde{H}_{h}^{(i)}(x)=U_{h}^{(i)}(x)-u_{n}^{(i)}(x), \quad x \in \Omega_{h}^{(2)} \cup \Omega_{h}^{(3)} .
$$

It follows that

$$
\left\|U_{h}^{(i)}-u_{h}^{(i)}\right\|_{h} \leqq C_{i}\left[\max _{\Omega_{h^{\prime}} \cup \Omega_{h}(s) \cup \Omega_{h}(s)}\left|U_{h}^{(i)}-u_{h}^{(i)}\right|+\left|\lambda_{h}^{(i)}-\lambda^{(i)}\right|+h^{4}\right] .
$$

Combining (8.13), (8.15), (8.16), and (8.17) yields the proof of Theorem 8.1.

Let us observe some simple consequences of Theorem 8.1. Since the $\lambda^{(i)}$ are real, we have

$$
\left|\operatorname{Re} \lambda_{h}^{(i)}-\lambda^{(i)}\right| \leqq C h^{4} .
$$

Also, when $\lambda^{(i)}$ is simple, $\lambda_{h}^{(i)}$ will be real for $h$ sufficiently small. This is because the matrix $\left[l_{h}(x, y)\right]_{x, y \in \Omega_{h}}$ is real. Thus, if $\lambda_{h}^{(i)}$ were complex, its conjugate $\left[\lambda_{h}^{(i)}\right]^{-}$would also be a distinct eigenvalue of $\Delta_{h}$ converging to $\lambda_{h}^{(i)}$. But this is impossible, since $\left[\lambda_{h}^{(i)}\right]^{-}$ must converge to some $\lambda^{(i)} \neq \lambda^{(i)}$.

We normalized $U_{h}^{(i)}$ by requiring $\left\|U_{h}^{(i)}\right\|_{h}=1$. This determines $U_{h}^{(i)}$ only up to a multiplicative constant of modulus 1 . If we specify this constant by requiring that $\left\langle U_{h}^{(i)}, V_{h}^{(i)}\right\rangle_{h}^{\prime} \geqq 0$, then when $\lambda^{(i)}$ is simple, $u_{h}^{(i)}$ is a real multiple of $u^{(i)}$, as can be seen from (8.9).

Theorem 8.1 shows that $U_{h}^{(i)}$ approximates to $O\left(h^{4}\right)$ an eigenfunction $u_{h}^{(i)}$ which depends on $h$. Properly normalized, however, $U_{h}^{(i)}$ will approximate to $O\left(h^{4}\right)$ an eigenfunction $u_{h}^{(i)}$ such that $\int_{a}\left|u_{h}^{(i)}\right|^{2} d x=1$, independently of $h$. In particular, when $\lambda^{(i)}$ is simple, $U_{h}^{(i)}$ will approximate the unique normalized eigenfunction $u^{(i)}$. This normalization is

$$
h^{2} \sum_{y \in \Omega_{h}} \alpha_{h}(y)\left|U_{h}^{(i)}(y)\right|^{2}=1,
$$

where $\alpha_{h}$ is given in the appendix of [6]. For a proof, see [6, Corollary 6.2].

9. Forced Vibration Problems. Let us remark that all of the results of the previous sections hold for the problem

$$
\Delta u(x)+(q(x)+\lambda) u(x)=0, \quad x \in \Omega, \quad u(x)=0, \quad x \in \partial \Omega,
$$


where $q$ is nonpositive and smooth on $\Omega$, and for the discrete Green's function $G_{h}$ defined by

$$
\left(\Delta_{h, x}+q(x)\right) G_{h}(x, y)=-h^{-2} \delta(x, y), \quad x, y \in \Omega_{h} .
$$

The proofs require only that the additional term $q$ be carried along throughout. We make this remark because we next wish to consider the problem

$$
\Delta u(x)+r(x) u(x)=F(x), \quad x \in \Omega, \quad u(x)=0, \quad x \in \partial \Omega,
$$

for $F$ and $r$ given smooth functions on $\Omega$. Problem (9.3) is a forced vibration problem and an $O\left(h^{2}\right)$ analogue of it was studied by Bramble in [1].

Let us rewrite (9.3) in the form

$$
\Delta u(x)+q(x) u(x)+\left(\sup _{\Omega} r\right) u(x)=F(x), \quad x \in \Omega, \quad u(x)=0, \quad x \in \partial \Omega,
$$

where $q(x) \equiv r(x)-\sup _{\Omega} r \leqq 0$ on $\Omega$. A unique solution $u$ of (9.3) or (9.4) exists if and only if sup $r$ is not an eigenvalue of the operator $\Delta+q$. Now, we consider the difference approximation

$$
\Delta_{h} U_{h}(x)+r(x) U_{h}(x)=F(x), \quad x \in \Omega_{h},
$$

where $\Delta_{h}$ is the difference operator defined in Section 2. We prove:

THEOREM 9.1. If (9.3) has a unique solution $u \in C^{6}(\bar{\Omega})$, there are constants $C, h_{0}$ such that for $h<h_{0},(9.5)$ has a unique solution $U_{h}$ for which

$$
\max _{\mathrm{B}_{h}}\left|U_{h}-u\right|<C h^{4} \text {. }
$$

Proof. Let $G_{h}$ be the discrete Green's function defined in (9.2). Then, for $x \in \Omega_{h}$,

$$
\begin{aligned}
\left|U_{h}(x)-u(x)\right| & =\left|h^{2} \sum_{y \in \Omega_{h}} G_{h}(x, y)\left[\Delta_{h} u(y)+q(y) u(y)-\Delta_{h} U_{h}(y)-q(y) U_{h}(y)\right]\right| \\
& \leqq \sup _{\boldsymbol{Q}}|q| h^{2} \sum_{y \in \Omega_{h}}\left|G_{h}(x, y)\right|\left|U_{h}(y)-u(y)\right|+h^{2} \sum_{y \in \Omega_{h}}\left|G_{h}(x, y)\right|\left|\tau_{h} u(y)\right| .
\end{aligned}
$$

Therefore, using (5.13) and (5.14) for $G_{\Lambda}$ of (9.2) and (2.5),

$$
\left|U_{\mathrm{L}}(x)-u(x)\right| \leqq C\left[h^{2} \sum_{y \in \Omega_{b}}\left|G_{h}(x, y)\right|\left|U_{h}(y)-u(y)\right|+h^{4}\right] \text {. }
$$

Employing (5.17), this yields

$$
\max _{\Omega_{b}(s) \cup \Omega_{k}(s)} \mid U_{k}-u_{i}^{\prime} \leqq C\left[k \max _{\varepsilon_{k}}\left|U_{k}-u\right|+k^{e}\right] \text { s }
$$

while $(5.16)$ and Schwarz's inequality yield

$$
\max _{\sigma_{2}} \mid \mathcal{C}_{k} \cdot u_{1}^{\prime} \leqq\left([]_{1}^{\prime} C_{k}=u_{1,}^{\prime} \cdot \because k^{2}\right]
$$

Irom $(9.7)$ and $(9.8)$, we sec

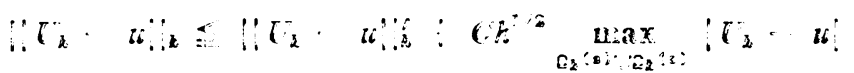

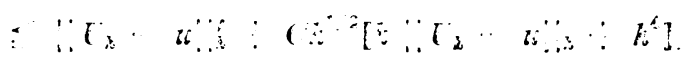


which implies

$$
\left\|U_{h}-u\right\|_{h} \leqq C\left[\left\|U_{h}-u\right\|_{h}^{\prime}+h^{4}\right] .
$$

Finally, we complete the proof by using Parseval's identity to estimate

$$
\left\|U_{h}-u\right\|_{h}^{\prime}=\left[\sum_{i}\left|\left\langle U_{h}-u, V_{h}^{(i)}\right\rangle_{h}^{\prime}\right|^{2}\right]^{1 / 2},
$$

where $V_{h}^{(i)}$ is the eigenvector associated with $\mu_{h}^{(i)}$ in the symmetric problem $\Delta_{h} V_{h}^{(i)}(x)+\left(q(x)+\mu_{h}^{(i)}\right) V_{h}^{(i)}(x)=0, \quad x \in \Omega_{h}^{\prime}, \quad V_{h}^{(i)}(x)=0, \quad x \in \Omega_{h}^{(2)} \cup \Omega_{h}^{(3)}$.

Define $H_{h}$ by

$\Delta_{h} H_{h}(x)+q(x) H_{h}(x)=0, \quad x \in \Omega_{h}^{\prime}, \quad H_{h}(x)=U_{h}(x)-u(x), x \in \Omega_{h}^{(2)} \cup \Omega_{h}^{(3)}$. From (5.21), we have

$$
\max _{\Omega_{h^{\prime}}}\left|H_{h}\right| \leqq C \max _{\Omega_{h}(z) \cup \Omega_{h}(3)}\left|U_{h}-u\right|,
$$

or, employing (9.7), (9.8), (9.9),

$$
\max _{\Omega_{h^{\prime}}}\left|H_{h}\right| \leqq C\left[h\left\|U_{h}-u\right\|_{h}^{\prime}+h^{4}\right] .
$$

Then, we have

$$
\begin{aligned}
\mu_{h}^{(i)}\left\langle U_{h}-u, V_{h}^{(i)}\right\rangle_{h}^{\prime} & =\left\langle H_{h}+u-U_{h},\left(\Delta_{h}+q\right) V_{h}^{(i)}\right\rangle_{h}^{\prime}+\mu_{h}^{(i)}\left\langle H_{h}, V_{h}^{(i)}\right\rangle_{h}^{\prime} \\
& =\left\langle\left(\Delta_{h}+q\right)\left(H_{h}+u-U_{h}\right), V_{h}^{(i)}\right\rangle_{h}^{\prime}+\mu_{h}^{(i)}\left\langle H_{h}, V_{h}^{(i)}\right\rangle_{h}^{\prime} \\
& =(\sup r)\left\langle U_{h}-u, V_{h}^{(i)}\right\rangle_{h}^{\prime}-\left\langle\tau_{h} u, V_{h}^{(i)}\right\rangle_{h}^{\prime}+\mu_{h}^{(i)}\left\langle H_{h}, V_{h}^{(i)}\right\rangle_{h}^{\prime} .
\end{aligned}
$$

Now, since sup $r$ is not an eigenvalue $\lambda^{(i)}$ of $\Delta+q$ and $\mu_{h}^{(i)} \rightarrow \lambda^{(i)}$ as $h \rightarrow 0$, there are constants $C, h_{0}$ such that for $h<h_{0}$,

$$
\max _{i}\left|\mu_{h}^{(i)}-\sup r\right|^{-1}<C, \quad \max _{i} \mu_{h}^{(i)} /\left|\mu_{h}^{(i)}-\sup r\right|<C,
$$

and so

$$
\left|\left\langle U_{h}-u, V_{h}^{(i)}\right\rangle_{h}^{\prime}\right| \leqq C\left[\left|\left\langle\tau_{h} u, V_{h}^{(i)}\right\rangle_{h}^{\prime}\right|+\left|\left\langle H_{h}, V_{h}^{(i)}\right\rangle_{h}^{\prime}\right|\right] .
$$

Using this in (9.10), we see that

$$
\left\|U_{k}-u\right\|_{h}^{\prime} \leqq C\left[\left\|\tau_{k} u\right\|_{h}^{\prime}+\left\|_{1}^{\prime} H_{h}\right\|_{h}^{\prime}\right] \leqq C\left[h^{4}+h\left\|_{1}^{\prime} \tau_{h}-u_{\|}^{\prime}\right\|_{h}^{\prime}\right]_{s}
$$

by (9.11), from which it follows that

$$
\because T_{k} \cdot u \|_{k} \leqq C h^{4}
$$

completing the proof.

I et us remark that by employing the results of $[6]$, the above technique of proof will show that a unique solution of the forced vibration problem:

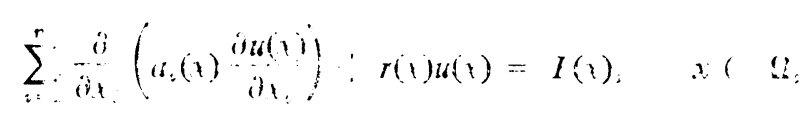

$$
\text { wi) }=0 . \quad x(30 .
$$


can be approximated to $O\left(h^{2}\right)$ by using the symmetric difference scheme given in [6] at the beginning of Section 7.

Applied Physics Laboratory

The Johns Hopkins University

Silver Spring, Maryland 20910

1. J. H. BRAmble, "Error estimates for difference methods in forced vibration problems," SIAM J. Numer. Anal., v. 3, 1966, no. 1, pp. 1-12. MR 34 \#969.

2. J. H. BRAMBLe \& B. E. HubBard, "New monotone type approximations for elliptic problems," Math. Comp., v. 18, 1964, pp. 349-367. MR 29 \#2982.

3. J. H. BRAMBLE \& V. C. THOMÉE, "Point-wise bounds for discrete Green's functions," SIAM J. Numer. Anal., v. 6, 1969, pp. 583-590.

4. R. Courant \& D. Hilbert, Methoden der Mathematischen Physik. Vol. I, Springer, Berlin, 1931; English transl., Interscience, New York, 1953. MR 16, 426.

5. B. E. HUBBARD, "Bounds for eigenvalues of the free and fixed membrane by finite difference methods," Pacific J. Math., v. 11, 1961, pp. 559-590. MR 25 \#4633.

6. J. R. KUTTLER, "Finite difference approximations for eigenvalues of uniformly elliptic operators," SIAM J. Numer. Anal., v. 7, 1970.

7. M. MARCUS \& H. Minc, A Survey of Matrix Theory and Matrix Inequalities, Allyn and Bacon, Boston, Mass., 1964. MR 29 \#112.

8. H. S. PRICE, "Monotone and oscillation matrices applied to finite difference approximations," Math. Comp., v. 22, 1968, pp. 489-516. MR 38 \#875.

9. H. F. WEINBERGER, "Lower bounds for higher eigenvalues by finite difference methods," Pacific J. Math., v. 8, 1958, pp. 339-368; erratum, 941. MR 21 \#6097. 